\title{
Ataxia induced by small amounts of alcohol
}

\author{
F Setta, J Jacquy, J Hildebrand, M-U Manto
}

\begin{abstract}
A patient is described who exhibited cerebellar ataxia after drinking small amounts of alcohol. Intake of $5 \mathrm{~g}$ alcohol induced a gaze evoked nystagmus, a scanning speech, a body sway after eye closure, and bilateral postural leg tremor. Kinematic and EMG analysis of fast wrist movements showed normal movements before and marked hypermetria after alcohol intake. Dysmetria was due to abnormal programming of antagonist muscle activity.

(F Neurol Neurosurg Psychiatry 1998;65:370-373)
\end{abstract}

Keywords: alcohol; cerebellum; hypermetria

Alcohol is a cause of late cortical cerebellar degeneration of the anterior lobe. ${ }^{1-3}$ These patients typically exhibit ataxia of the lower limbs, ataxia of gait, and trunk instability. Less frequent clinical findings include nystagmus, dysarthria and upper limb incoordination. ${ }^{2}{ }^{3}$ The neuropathological changes involve in particular Purkinje cells at the level of the anterior and superior vermis, and paravermal parts of the anterior lobe in more advanced cases. ${ }^{1}$ It has been shown that alcoholic patients exhibiting cerebellar ataxia do not have higher alcohol consumption than non-ataxic alcoholic patients, suggesting that alcohol induced cerebellar degeneration might be due to an idiosyncratic sensitivity to alcohol. ${ }^{4}$ We report on a patient with a normal neurological examination who showed a high vulnerability to small doses of alcohol. Small quantities of alcohol induced a cerebellar syndrome with characteristics reminiscent of a cerebellar cortical atrophy of the anterior lobe.

Service de Neurologie, Hôpital Erasme-ULB, Bruxelles, Belgique

J Hildebrand

M-U Manto

Correspondence to: Dr Mario-Ubaldo Manto, Service de Neurologie, Fonds National de la Recherche Scientifique, Hôpital Erasme-ULB, 808 Route de Lennik, 1070 Bruxelles, Belgium.

Telephone 003225556747 ; fax 003225553942 .

Received 13 August 1997 and in final revised form 19 February 1998

Accepted 24 February 1998 following blood tests were normal: sedimentation rate, glucose concentration, liver function tests, ammonia concentration, and red blood cell transketolase (an index of thiamine nutritional status ${ }^{4}$ ), vitamin $\mathrm{E}$ concentration. Investigations for a cardiomyopathy or an occult malignancy were negative. Motor and sensory nerve conduction velocities in his lower limbs were also normal. He was given thiamine (600 $\mathrm{mg} /$ day) for 5 months with no effect on the alcohol induced ataxia.

We examined this patient for the first time in 1997. He wanted to drink wine occasionally. There was no family history of neurological disease or enhanced susceptibility to alcohol intake (his father and his two brothers drank from 10 to $50 \mathrm{~g}$ alcohol a day for more than 10 years without complaints). General physical examination was unremarkable. There was no postural hypotension. Neurological examination showed normal mental status (mini mental state examination (MMSE) 29/30). There was no scanning speech, and finger-to-nose test, fine finger movements, alternate movements of the hands, heel-to-knee test, Romberg's test, and tandem gait were all normal. Strength and careful sensory examination were unremarkable. Tendon reflexes were brisk and plantar reflexes were flexor. The following blood studies were normal: blood cell count, renal and liver function tests, albumin concentration, lipids, uricaemia, antinuclear antibodies, concentrations of vitamins B12 and folic acid, and thyroid function tests. No alcohol was detected in his blood. Sensory evoked potentials (all four limbs), auditory evoked potentials, motor evoked potentials in the upper limbs, motor and sensory conduction velocities in the upper and lower limbs, and EEG were all within the normal range. Brain CT, brain MRI, and single photon emission computed tomography ( ${ }^{99 \mathrm{~m}}$ Tc-HMPAO) were normal. Brain PET was not performed.

With informed consent, we tested the effects of $5 \mathrm{~g}$ alcohol given orally on neurological examination (20 minutes after alcohol intake) and on fast wrist flexion movements (30 minutes after alcohol intake) in a control group of five right handed healthy men (mean age 42 (SD 5) years) and in our patient. In the control group, this small dose of alcohol did not modify the results of neurological examination. By contrast, in our patient, movements in his upper limbs were irregular after alcohol consumption, and we found a gaze evoked nys- 
A

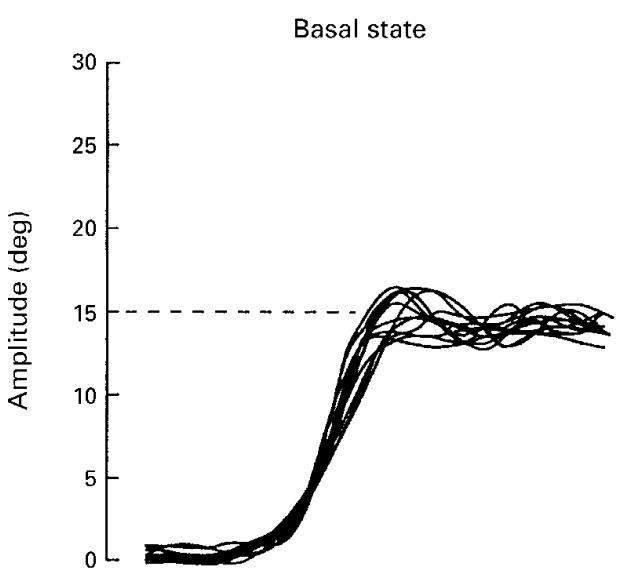

Wrist flexion movements

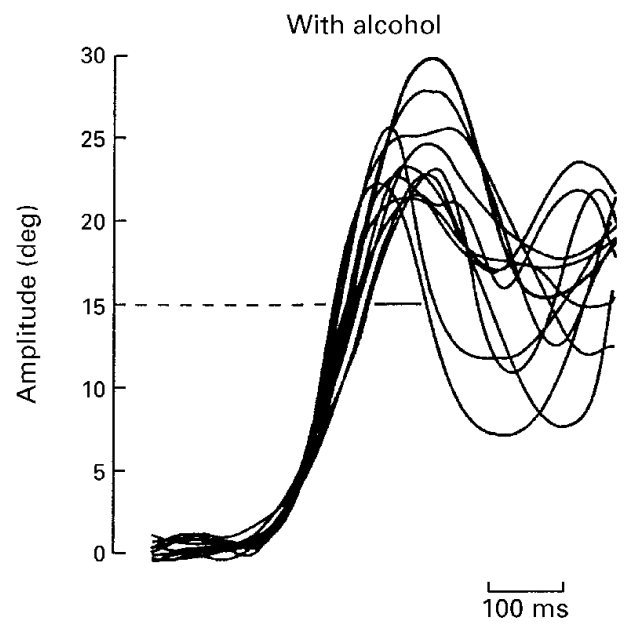

B

EMG activities

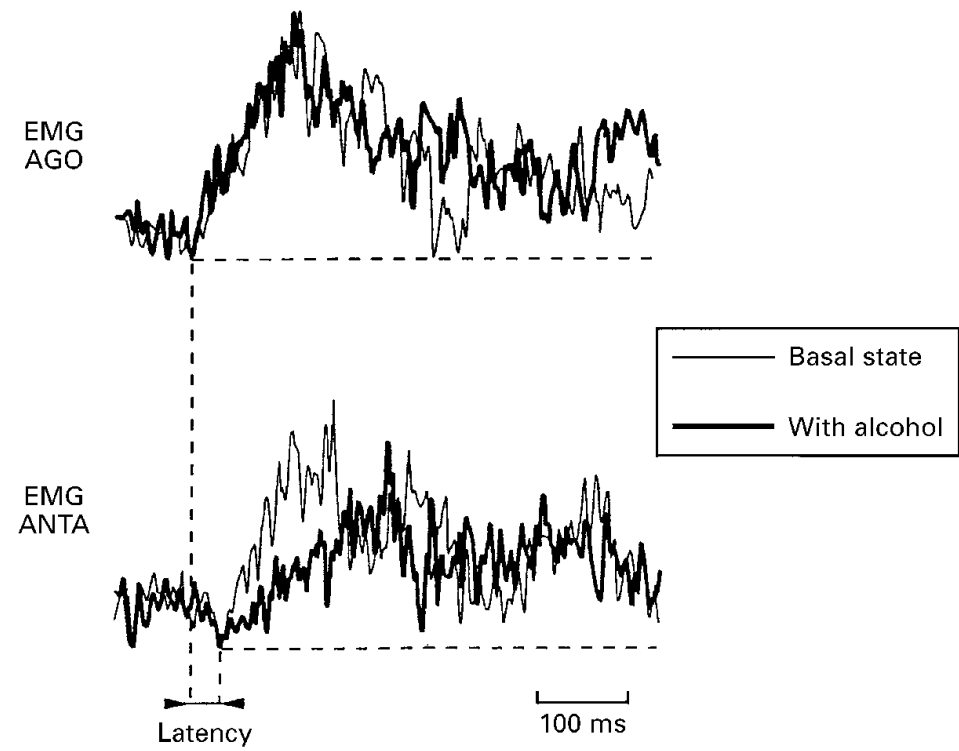

(A) Kinematic features of fast and accurate right wrist flexions performed by the patient before (left part) and after alcohol intake (right part). Each panel corresponds to the superimposition of the individual records of position for 12 flexion movements. The aimed target is located at $15^{\circ}$ from the start position. (B) Electromyographic features of fast flexions for right wrist before and after alcohol intake in our patient. Superimposition of the rectified and averaged agonist EMG activities (EMG AGO; top panel) and of the rectified and averaged antagonist EMG activities (EMG ANTA; bottom panel), before (thin traces) and after alcohol consumption (thick traces). The number of wrist flexions is 12.

tagmus and a mild scanning speech. Romberg's test showed marked anterior and posterior oscillations after eye closure. A low frequency bilateral postural leg tremor with a waxing and waning amplitude and which predominated on the right side was present. Tandem gait required one aid. Strength and sensory examination were normal, tendon reflexes were unchanged, and plantar reflexes remained flexor.

We analysed kinematics and EMG indices of agonist (flexor carpi radialis) and antagonist (extensor carpi radialis) activities associated with fast wrist flexion movements before (basal condition) and after intake of alcohol, using a method previously described. ${ }^{5}$ Experimental conditions before and after alcohol intake were identical. The patient and healthy subjects were comfortably seated with the forearm wrapped on to the arm of an armchair. Palm and fingers were wrapped on to a rigid light plate to avoid unwanted rotations of joints other than the wrist. Computerised motion analysis in three dimensions was made with a Selspot II system (Selcom, Sweden). One infrared light emitting diode was attached to the forefinger. Sampling rate was 300/s. Velocity curves were obtained after digital differentiation of the position signal. The aimed target was located $15^{\circ}$ away from the starting position and we recorded 12 movements in both conditions. The EMG signals were amplified, filtered (2000×; 30-8000 Hz), full wave rectified, and averaged. 


\section{Results}

In the control group, mean movement amplitude was 15.3 (SD 0.9) ${ }^{\circ}$ before and was 14.9 (SD 1.0) ${ }^{\circ}$ after alcohol intake (Student's $t$ test; $\mathrm{p}=0.61$ ). The mean peak velocity was 597 (SD $35) \%$ before and 588 (SD 42)\% after alcohol intake (Student's $t$ test: $\mathrm{p}=0.52$ ). The mean onset latency of the antagonist activity was 51 (SD 12) $\mathrm{ms}$ before and was 46 (SD 15) $\mathrm{ms}$ with alcohol (Student's $t$ test: $\mathrm{p}=0.64$ ). The mean ratio of the rate of rise of agonist activity (AGO Q30) divided by the rate of rise of antagonist activity (ANTA Q30) was 0.97 (SD 0.09) in the basal state and was 0.97 (SD 0.09) with alcohol (Student's $t$ test: $\mathrm{p}=0.70$ ). The figure (A) illustrates the flexion movements of the right wrist in our patient before and after alcohol intake. Mean movement amplitude was $14.5^{\circ}$ and mean peak velocity was $570 \%$ in the basal state. With alcohol, movements were clearly hypermetric: the mean movement amplitude was $24.3^{\circ}$, and mean peak velocity was $579 \%$ s. The onset latency of the antagonist EMG activity was $33 \mathrm{~ms}$ before and $31 \mathrm{~ms}$ after alcohol intake. As illustrated in the figure (B), the rate of rise of antagonist activity was dramatically reduced after alcohol intake. The ratio of AGO Q30/ANTA Q30 changed from 1.09 to 2.51 . Review of individual traces of antagonist EMG activities obtained in our patient before alcohol intake showed that two components were demarcated in half of the 12 trials. The first component began about $30 \mathrm{~ms}$ after onset of the agonist burst and presented a peak of activity about $100 \mathrm{~ms}$ after the onset of antagonist activity. The second component was more independent, with a peak of activity occurring about $200 \mathrm{~ms}$ after onset of antagonist activity. After alcohol intake, we could not identify the first component.

We also measured the ethanol concentration in blood 90 minutes after alcohol intake in our patient. Although ataxic signs were still present, no ethanol was detected, suggesting a normal activity of hepatic alcohol dehydrogenase, an enzyme which clears moderate doses of ethanol from the blood in about 1 hour. ${ }^{6}$

\section{Discussion}

Our case illustrates the high vulnerability of the cerebellum to small amounts of alcohol in a patient with an otherwise normal neurological examination. Other patients with such a susceptibility to alcohol have not been described. After alcohol intake, neurological signs were mainly suggestive of a cerebellar cortical atrophy of the anterior lobe. ${ }^{2}{ }^{7}$ Indeed, a low frequency postural leg tremor during sustained posture was present and eye closure induced a body sway in the anterior-posterior plane. ${ }^{7}$ Moreover, the kinematics and the dissociation between agonist and antagonist EMG activities were strongly in favour of a disruption of cerebellar pathways. ${ }^{58}$

What is the interpretation of hypermetria in our patient? It might be due to an inability to tune the rate of rise of antagonist activity, or might be the consequence of the loss of an initial component of antagonist EMG. The analysis of the ratio AGO Q30/ANTA Q30 suggested that rapid voluntary movements were hypermetric due to the inability to generate a sufficient rate of rise of the antagonist activity. Such a cerebellar hypermetria associated with an inability to develop a normal rate of rise of the antagonist activity, despite adequate timing between agonist and antagonist muscles, was described initially in patients with idiopathic cerebellar degeneration involving vermal and paravermal parts of the cerebellum. ${ }^{8}$ To our knowledge, the reduced rate of rise of antagonist activity has not been shown in extracerebellar disorders. The defect in the tuning of the shape of the antagonist activity ${ }^{8}$ in the present case indicated that the spinocerebellum was probably the main site of the deleterious action of alcohol and its metabolites ${ }^{9}$ on cellular and synaptic activity. Alternatively, alcohol could have impaired activity of afferent spinocerebellar tracts or efferent projections from the spinocerebellum. Moreover, inspection of individual traces of antagonist EMG activities in our patient showed that two components were identified in six of the 12 trials before alcohol intake. Thus, the change in EMG pattern after alcohol intake could be described as a loss of the first component rather than a change in the rate of rise of the antagonist. This hypothesis is in agreement with the findings of Wild and Corcos who have suggested that cerebellar hypermetria might be due to the reduction in an early component of the antagonist. ${ }^{10}$ However, the distinction in two components of the antagonist EMG could not be made in half of the trials, and the peak amplitude of the first component (when the two components were identified) was higher than the peak value of the second component, unlike the findings of Wild and Corcos. ${ }^{10}$ In the trials obtained in patients with vermal and paravermal atrophy, we could not distinguish two components in antagonist activity. ${ }^{8}$ Finally, the possibility of alcohol related impairment of neuronal activity of the medial portions of the frontal lobes seems unlikely, but cannot be excluded. ${ }^{9}$

It should be highlighted that hypermetria can be seen in healthy subjects in absence of deficit of antagonist activity. ${ }^{11}$ This physiological hypermetria is characterised by a normal adaptation to increased inertia: when a mass is added to the moving hand, the hypermetria of fast movements turns into normometria. Moreover, hypermetria can be seen in cerebellar patients in the absence of too late or too little antagonist activity. Indeed, even when wrist movements are considered, many muscles throughout the limb other than prime movers are active physiologically. ${ }^{12}{ }^{13}$ Abnormal control of activity of synergic muscles contributes to cerebellar dysmetria.

Ethanol might have induced cerebellar ataxia in our patient by its effects on $\gamma$-aminobutyric acid (GABA), N-methyl-D-aspartate (NMDA), or serotonin receptors. ${ }^{9}$ GABA is a major neurotransmitter in the cerebellum, and ethanol strongly acts on GABA-A receptors, modulating chloride flux. ${ }^{9}$ However, although the effects of alcohol on the CNS are better understood and a genetic inheritance might play a determinant part, it is still not clear why the spinocerebellum 
is selectively vulnerable. ${ }^{9}$ The recent finding that medial parts of the cerebellum are also preferentially injured in cases of high pyrexia ${ }^{714}$ suggests a common mechanism generating ataxia due to alcohol or hyperpyrexia.

This work was supported by a grant from the Belgian National Research Foundation.

1 Victor M, Adams RD, Mancall EL. A restricted form of cerebellar cortical degeneration occurring in alcoholic patients. Arch Neurol 1959;1:579-68.

2 Mauritz KH, Dichgans J, Hufschmidt A. Quantitative analysis of stance in late cortical cerebellar atrophy of the anterior lobe and other forms of cerebellar ataxia. Brain 1979;102:461-82.

3 Johnson-Greene D, Adams KM, Gilman S, et al. Impaired upper limb coordination in alcoholic cerebellar degeneraupper limb coordination in alco

4 Estrin WJ. Alcoholic cerebellar degeneration is not a dose-dependent phenomenon. Alcohol Clin Exp Res 1987;

5 Manto M, Jacquy J, Hildebrand J, et al. Recovery of hypermetria after a cerebellar stroke occurs as a multistage process. Ann Neurol 1995;38:437-45.
6 Wyngaarden JB, Smith LH. Cecil textbook of medicine. Philadelphia: Saunders, 1988:49.

7 Manto M, Topka H. Reversible cerebellar gait ataxia with postural tremor during episodes of high pyrexia. Clin Neurol Neurosurg 1996;98:227-30.

8 Manto M, Godaux E, Jacquy J, et al. Cerebellar hypermetria associated with a selective decrease in the rate of rise of antagonist activity. Ann Neurol 1996;39:271-4.

9 Gilman S, Koeppe RA, Adams K, et al. Positron emission tomographic studies of cerebral benzodiazepine-receptor binding in chronic alcoholics. Ann Neurol 1996;40:163-71.

10 Wild B, Corcos DM. Cerebellar hypermetria: reduction in the early component of the antagonist electromyogram. Mov Disord 1997;12:604-7.

11 Manto M, Godaux E, Jacquy J. Cerebellar hypermetria is larger when the inertial load is artificially increased. Ann Neurol 1994;35:45-52.

12 Manto M. Pathophysiology of cerebellar dysmetria: the imbalance between the agonist and the antagonist electromyographic activities. Eur Neurol 1996;36:333-7.

13 Thach WT, Perry JG, Kane SA, et al. Cerebellar nuclei: rapid alternating movement, motor somatotopy, and a mechanism for the control of muscle synergy. Rev Neurol 1993;149:607-28.

14 Manto M, Goldman S, Hildebrand J. Cerebellar gait ataxia following neuroleptic malignant syndrome. F Neurol 1996; 243:101-2.

\section{NEUROLOGICAL STAMP}

\section{Jean Alfred Fournier (1832-1914)}

Jean Alfred Fournier, Professor of Dermatology at the University of Paris and Director of the internationally famous venereal hospital at the Hospital of St Louis, wrote extensively on the clinical and social aspects of this subject. Fournier introduced the concept of "parasyphilis" (tabes dorsalis and general paresis) and was the first to point out the relation of syphilis to these disorders. He also emphasised the importance of congenital syphilitic disease and wrote on its social aspects (Syphilis et Marriage 1890). In 1901 he formed the Society for Moral and Sanitary Prophylaxis which carried on an educational programme to control venereal diseases in France. He also had a view about the cause of epilepsy and wrote "one of the nervous afflictions which onanism occasions most frequently is epilepsy.... There are very few physicians who have not observed where it has been produced, maintained or aggravated by the practice of this pernicious habit".

In 1946 France issued a stamp to raise funds for the fight against venereal disease (Stanley Gibbons 960, Scott B201).

L F HAAS

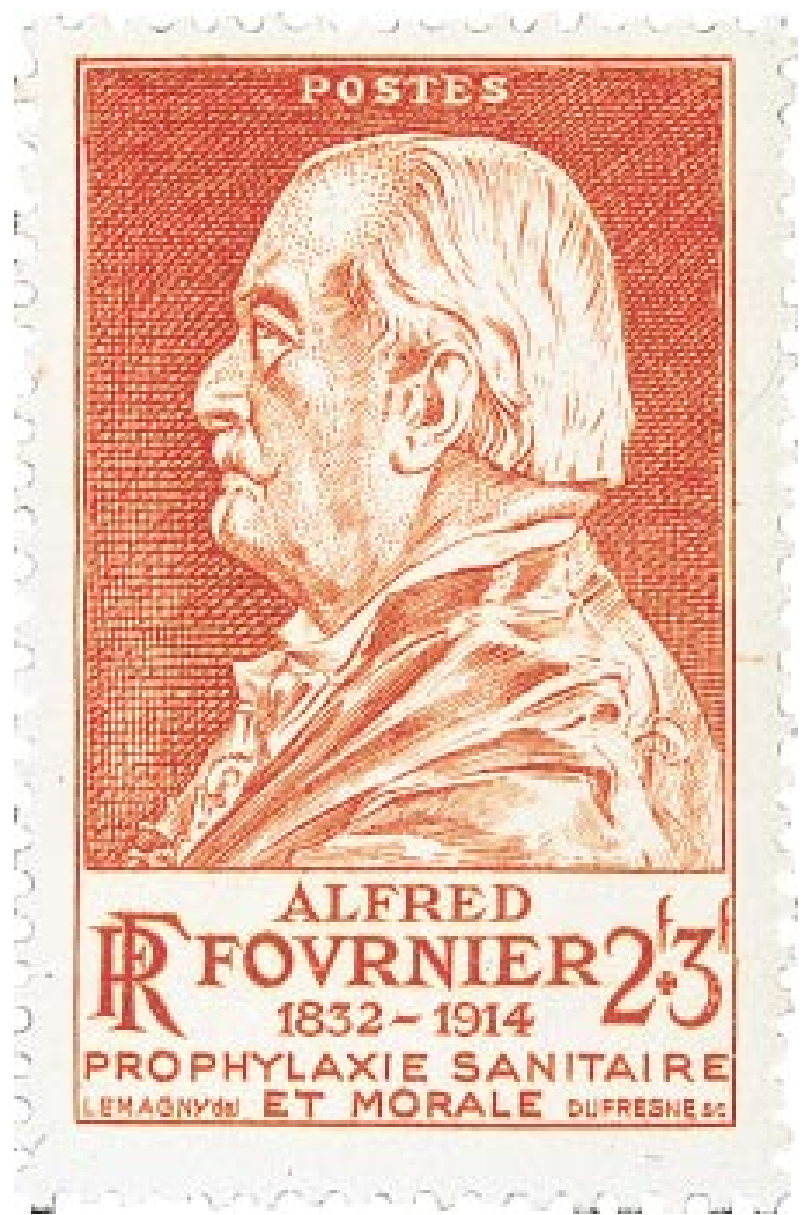

